infection for testing antibiotics that utilize free time above MIC as the pharmacokinetic (PK) driver to predict efficacy. We further established the mouse model for antibiotic testing by defining the in vivo efficacy of ciprofloxacin (CIP), an antibiotic that uses the free area under the curve over MIC (fAUC/MIC).

Methods Lower genital tract infection with $\mathrm{Ng}$ strain FA1090 was established in female mice using published methods for two days, after which increasing oral doses of CIP (or controls) were administered ( $\mathrm{n}=10-20 \mathrm{mice} /$ group) and infection was quantified for 8 days. Plasma drug levels from uninfected mice were measured after administration of similar doses of CIP, and PK parameters (modeled using WinNonlin software) were correlated with observed efficacy.

Results Single oral doses ranging from 5 to $60 \mathrm{mg} / \mathrm{kg}$ CIP showed significant activity against strain FA1090, with the highest doses $(15,30$, and $60 \mathrm{mg} / \mathrm{kg}$ ) clearing $100 \%$ of infections within 8 days; these correspond to predicted fAUC/MICs of $66-264$. The $60 \mathrm{mg} / \mathrm{kg}$ dose cleared infection in all mice within $48 \mathrm{~h}$, which we defined previously as the endpoint in the model that best correlates with in vivo exposures required for successful CRO/CFX treatment regimens.

Conclusion The gonorrhea mouse model shows a dose-dependent response for CIP against a $\mathrm{CIP}^{\mathrm{S}}$ strain with a dose of 60 $\mathrm{mg} / \mathrm{kg}$ required to clear infection in $48 \mathrm{hrs}$. PK modeling suggests that achieving exposures necessary for effective treatment of CIPR strains (mic $\geq 1 \mu \mathrm{g} / \mathrm{ml}$ ) would be challenging. These data that establish PK/PD correlations for CIP - with a fAUC/ MIC driver- further strengthens the usefulness of this mouse model to test novel antimicrobial compounds against gonorrhea.

Disclosure No significant relationships.

\section{P691 WIDESPREAD USE OF HIGH-DOSE CEFTRIAXONE THERAPY FOR UNCOMPLICATED GONORRHEA WITHOUT REPORTED CEFTRIAXONE TREATMENT FAILURE}

\begin{abstract}
${ }^{1}$ Yan Han*, ${ }^{2}$ Yueping Yin, ${ }^{2}$ Shaochun Chen, ${ }^{3}$ Xiang-Sheng Chen, ${ }^{4}$ Jun Liu. ${ }^{1}$ National Center for STD Control, Chinese Center for Disease Control and Prevention, Institute of Dermatology, Chinese Academy of Medical Sciences and Peking Union Medical College, Reference Laboratory, Nanjing, China; ${ }^{2}$ National Center for STD Control, China CDC Institute of Dermatology Chinese Academy of Medical Sciences and Peking Union Medical College, Reference Lab, Nanjing, China; ${ }^{3}$ National Center for STD Control, Chinese Center for Disease Control and Prevention, Nanjing, China; ${ }^{4}$ Massachusetts General Hospital, Harvard Medical School, Cambridge, USA
\end{abstract}

\subsection{6/sextrans-2019-sti.757}

Background Antimicrobial resistance (AMR) to N. gonorrhoeae has emerged for each of the antibiotics following their introduction into clinical practice recommended as first-line therapies. To improve rational and effective clinical antibiotic treatment, we analyzed the prescription patterns of antibiotics and its therapeutic effect in the treatment of uncomplicated gonorrhea in China.

Methods We obtained data from a follow-up multicenter-surveillance program. Multinomial logistic regression analyses were conducted to explore the associations between demographic/clinical variables with the levels of sensitivity to ceftriaxone and prescription of high-dose ceftriaxone.

Results In this study, 1686 patients infected with N. gonorrhoeae were recruited in a surveillance network during the period of 1 January 2013 through 31 December 2017 in 7 hospitals distributed in 5 provinces. The prevalence of isolates with decreased susceptibility to ceftriaxone was 9.8\% (131/ 1333), fluctuating between $5.6 \% \sim 12.1 \%$. Injectable ceftriaxone was chosen as the first-line treatment among $83.1 \%$ patients, and most of them $(72.7 \%, 1018 / 1401)$ received more than $1000 \mathrm{mg}$ dosage. Patients who were infected with gonorrhea or infected with other STDs before (AOR 1.611 95\%CI [1.103-2.352]; AOR 2.329 95\%CI [1.553-3.494]) or who used already antibiotics for this infection (AOR 1.597, 95\%CI [1.04-2.452]) were associated with higher prescribed ceftriaxone dosage prescribed. All of the patients recruited in this study were cured regardless of the isolates' susceptibility to ceftriaxone or the dosage of ceftriaxone they received.

Conclusion No ceftriaxone failure treatment for uncomplicated gonorrhea were reported in China, however, high-dose ceftriaxone were widely used in China, its impacts needs further studies.

Disclosure No significant relationships.

\section{P692 GENTAMICIN SUSCEPTIBILITY TO NEISSERIA GONORRHOEAE IN MALAWI AFTER TWENTY-FIVE YEARS OF SUSTAINED USE}

${ }^{1} J a n e$ Chen*, ${ }^{2}$ Mitch Matoga, ${ }^{2}$ Cecilia Massa, ${ }^{2}$ Beatrice Ndalama, ${ }^{2}$ Edward Jere, ${ }^{3}$ Robert Krysiak, ${ }^{4}$ Tarsizio Chikaonda, ${ }^{5}$ Marcia Hobbs, ${ }^{3}$ Myron Cohen, ${ }^{3}$ Irving Hoffman. 'University of North Carolina at Chapel Hill, Epidemiology, Chapel Hill, USA; ${ }^{2}$ UNC Project Malawi, Lilongwe, Malawi; ${ }^{3}$ University of North Carolina at Chapel Hill, Division of Infectious Diseases, Chapel Hill, USA; ${ }^{4}$ UNC Project Lilongwe, Laboratory, Lilongwe, Malawi; ${ }^{5}$ University of North Carolina at Chapel Hill, Microbiology and Immunology, Chapel Hill, USA

\subsection{6/sextrans-2019-sti.758}

Background Gentamicin has been used exclusively for the treatment of Neisseria gonorrhoeae (GC) in Malawi, since 1993. Previous gentamicin susceptibility testing in 1993, 1996 and 2007 , showed $\geq 95 \%$ susceptibility by both agar dilution and E-test. However, clinical cure rates 1-2 weeks following treatment, have been in the $90 \%$ range. We are in the process of repeating this assessment to inform treatment guidelines.

Methods We are enrolling HIV-infected men presenting with acute urethritis at the sexually transmitted infections (STI) clinic at Bwaila District Hospital in Lilongwe, Malawi. All participants provide urethral swabs for STI etiologic testing, and are treated syndromically per Malawian standard of care, with gentamicin $240 \mathrm{mg}$ IM, doxycycline $100 \mathrm{mg}$, BID for 7 days, and metronidazole $2 \mathrm{~g}$ single dose. Patients are seen one week post-treatment for repeat clinical exam and GC culture. All specimens with a positive GC culture are tested locally for gentamicin susceptibility via E-test. E-test inhibition with ranges from $0-4,4-16$, and $\geq 16$ are categorized as high, moderate, and low susceptibility, respectively. Clinical cure is determined by genital examination.

Results 42 men with gonococcal urethritis have been enrolled to date. Baseline gentamicin E-test results: high susceptibility: 0-1: $21 \% ; 1-2$ : 60\%; 2-4: 19\%; moderate or low susceptibility $\geq 4$ : $0 \%$. $37 / 42$ men (88\%) returned for follow-up. 4/37 (11\%) were culture positive for GC, including 2 (5\%) symptomatic men. 3/4 (75\%) of the week one E-test results were in the high susceptibility range, and $1 / 4(25 \%)$ was in the low susceptibility range $(\geq 16)$. 